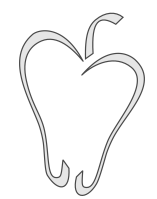

ISSN $1852-4249$

\title{
Commentary on "Granular discharge rate for submerged hoppers"
}

\author{
L. Staron ${ }^{1,2 *}$
}

\section{Introduction}

The paper by Wilson et al [1] describes experimental results on the discharge of hoppers filled with granular material and immersed in water. The discharge of dry granular matter through hoppers (as well as pipes and silos of various geometries) has been - and is still - much studied, due to the practical importance of these flow geometries but also because of the theoretical difficulties posed by their puzzling behavior. In this paper, the authors examine how the well-known results for "dry" hoppers are affected when the whole system is immersed in water. This experimental setup is original and discloses an intriguing new behavior. The authors have no clear physical explanation for this intriguing new behavior; however, the results are very interesting and leave many questions open for future research. Some of them are the subjects of the comments below.

\section{Scaling for the discharge velocity for dry hoppers}

In the introduction, the Beverloo scaling, that is the scaling for the discharge velocity for dry granu-

\footnotetext{
*E-mail: lydie.staron@upmc.fr

1 CNRS - Université Pierre et Marie Curie Paris 6, UMR 7190, Institut Jean Le Rond d'Alembert, F-75005 Paris, France.

2 School of Earth Sciences, University of Bristol, Queens Road, Bristol BR8 1RJ, United Kingdom.
}

lar hoppers, is understood as resulting from a freefall arch phenomenology (as described in [2]): force chains form and break intermittently above the orifice, shielding the grains from the pressure above and allowing free-fall dynamics. As a result, the velocity scales like $\sqrt{g D}$, where $D$ is the diameter of the orifice and $\mathrm{g}$ is gravity. This mechanism is described as intuitive. Yet, I find that the discrete (i.e., explicitly granular) picture of force chains is difficult to conciliate with the observation of stationary flow. Would not the intermittent formation and breaking of force chains lead to an intermittent flow regime, as observed for small apertures? How do force chains form over large apertures, up to 300 diameters in the present paper? This aspect is indeed central for the understanding of the physics of granular hoppers. An alternative interpretation of the discharge velocity is to consider the granular flow as a continuum with yield stress properties. These yield stress properties are the cause of the existence of "dead zones", namely areas of static equilibrium in the bottom corners of the containers and at the container's walls [3]. These "dead zones" can be seen themselves like solid walls surrounding the flow around the outlet. Considering this simplification of the dynamics of the grains above the outlet, one can again try to understand the discharge rate as resulting from the friction forces. I fully agree with the authors that the Janssen analysis applied to the walls of the container cannot account for the Beverloo scaling, and that a local argument is needed [4]. Interestingly, the Janssen analysis applied to the conduit formed by the static areas is a local argument that may yield the correct 
PAPers in Physics, vol. 6, ART. 060010 (2014) / L. Staron

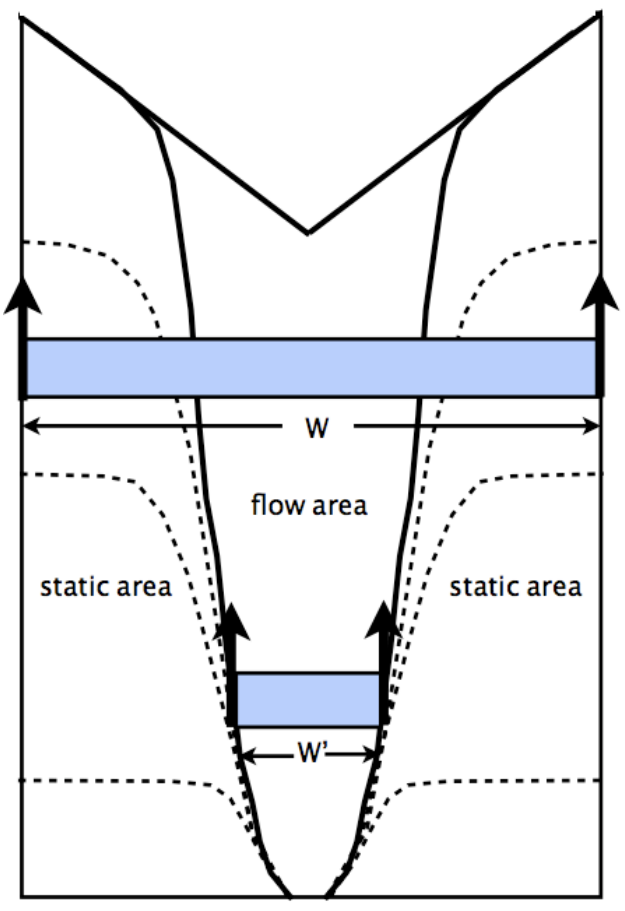

Figure 1: Illustration of a granular flow in a "dry" hopper. The static area forms solid walls surrounding the flow area. The classical Janssen analysis considers a slice of material spanning the whole hopper and the friction forces at the wall. Alternatively, the analysis may be performed for a slice of material spanning the flow area only, considering the friction forces acting in the bulk at the boundary between flow and static areas. (The dotted lines show typical streamlines.)

scaling. This is illustrated in Fig. 1. Equilibrating the pressure gradient and the friction forces at the walls for a slice of material spanning the whole container gives a saturation pressure scaling like $\rho g W / 2 \mu$, where $W$ is the hopper diameter and $\mu$ the coefficient of friction at the walls: this is the classical Janssen result. Using the same analysis, equilibrating the pressure gradient and the friction forces acting between the flow and the static areas for a slice of material spanning the flow only gives a saturation pressure scaling like $\rho g W^{\prime} / 2 \mu^{\prime}$, where $W^{\prime}$ is the flow width and $\mu^{\prime}$ the coefficient of friction at this location. Very likely, $W^{\prime}$ will scale like the outlet diameter $D$, hence the Beverloo scaling.

\section{Tall and short columns for im- mersed hoppers}

In the case of immersed hoppers, the authors successfully explain the new scaling for the discharge rate by replacing the free-fall-like velocity observed in dry cases by the Stokes velocity. This works well but, as stated by the authors, this probably gives an oversimplified picture of what really happens in the hopper above the outlet. In particular, since the grains initially filling the hoppers are arranged in dense packing, the flow at the aperture, necessary involving shearing, may induce locally dilation and the sucking of water in the hopper, as it is the case in quick sands for instance. In [5], the pressure at the bottom of dense granular columns immersed in water at the onset of collapsing was found to be negative. Of course the systems are different, but the same mechanism may apply. The experimental setup used in Wilson et al, being 3D and opaque, does not allow to observe in details what happens during the transient, when the flow starts. Hence, one can only speculate that probably dilation occurs, and probably it does affect the discharge rate. The shearing occurring when the flow starts (between the flow conduit and the surrounding static areas as illustrated in Fig. 1) is massive as it involves the full height of the column. One thus expects a non negligible quantity of fluid to be sucked into the hopper, and as a result, a change in the structure of the packing to occur. This may be at the origin of the difference between very tall column and shorter columns described by Wilson et al. The fluid sucked in shorter columns may be sufficient to affect the whole packing structure, while the structure of taller column may be partly preserved (the upper part for instance) so that the well-known dry granular behavior is preserved too. In other words, taller columns would still coincide with the discharge of dense granular flow, while shorter columns may imply the discharge of a dense suspension. This, of course, is only a supposition.

\section{Conclusion}

The experiment described in this paper is not easy to understand: the hopper configuration is complex in itself, and the fact that the system is immersed implies that we are at the frontier between 
Papers in Physics, vol. 6, ART. 060010 (2014) / L. Staron

the physics of dense dry granular packings and dense suspensions. Many interesting open questions remain, and certainly the work in progress by the same team will bring original new material to understand what happens during the discharge of granular flows - be it dry or immersed.

[1] T J Wilson, C R Pfeifer, N Meysingier, D J Durian, Granular discharge rate for submerged hoppers, Pap. Phys. 6, 060009 (2014).

[2] J E Hilton, P W Cleary, Granular flow during hopper discharge, Phys. Rev. E 84, 011307 (2011).
[3] L Staron, P-Y Lagrée, S Popinet, The granular silo as a continuum plastic flow: The hourglass vs the clepsydra, Phys. Fluids 24, 113303 (2012).

[4] H A Janssen, Versuche uber getreidedruck in silozelen, Zeitschr. Vereines Deutsch. Ing. 39, 1045 (1895).

[5] L Rondon, O Pouliquen, P Aussillous, Granular collapse in a fluid: Role of the initial volume fraction, Phys. Fluids 23, 073301 (2011). 